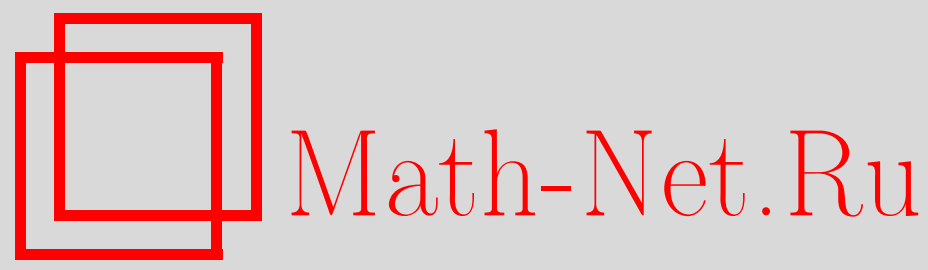

В. В. Рыжиков, О когомологичности коциклов, отвечающих эргодическим косым произведениям, Функи. анализ и его прил., 1996, том 30, выпуск $1,84-86$

DOI: https://doi.org/10.4213/faa514

Использование Общероссийского математического портала MathNet.Ru подразумевает, что вы прочитали и согласны с пользовательским соглашением

http://www . mathnet.ru/rus/agreement

Параметры загрузки:

IP : 3.81 .55 .215

26 апреля 2023 г., 07:53:23

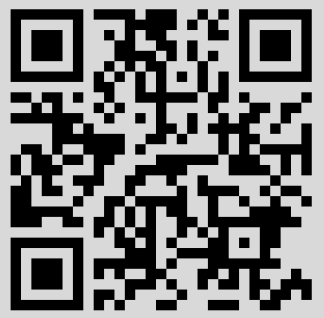




\title{
О когомологичности коциклов, отвечающих эргодическим косым произведениям
}

\author{
(c) $1996 . \quad$ В. В. Рыжиков ${ }^{1}$
}

Пусть $(X, \mathscr{B}, \mu)$ - пространство Лебега, $\mu(X)=1$, а $\mathscr{B}$ есть $\sigma$-алгебра всех классов $\mu$-измеримых множеств (два множества принадлежат одному классу, если различаются на множество меры 0). Обозначим через $\operatorname{Aut}(\mu)$ группу всех сохраняющих меру обратимых преобразований пространства $X$, которые также называются автоморфизмами. Пусть фиксированы $S \in \operatorname{Aut}(\mu)$ и семейство автоморфизмов $\left\{T_{x}: x \in X\right\}$, измеримо зависящее от $X$. Отображение $R:(X \times Y) \rightarrow$ $(X \times Y)$, определенное при $X=Y$ равенством $R(x, y)=\left(S(x), T_{x}(y)\right)$, называется косым произведением над $S$. Будем обозначать такое отображение $R$ через $S \times\left\{T_{x}\right\}$. Косому произведению $R$ отвечает коцикл $\Phi$ со значениями в $\operatorname{Aut}(\mu)$ : $\Phi(x, n)=T_{S^{n-1}(x)} \cdots T_{S(x)} T_{x}$ (общее определение коцикла см. в [1]).

Косые произведения $R=S \times\left\{T_{x}\right\}$ и $\widetilde{R}=S \times\left\{\widetilde{T}_{x}\right\}$ будем называть эквивалентными, если для некоторого измеримого семейства автоморфизмов $\left\{J_{x}: x \in X\right\}$ выполнено условие $\widetilde{T}_{x}=J_{S(x)}^{-1} T_{x} J_{x}$. Эквивалентные косые произведения метрически изоморфны, а отвечающие им коциклы когомологичны, т. е.

$$
\widetilde{\Phi}(x, n)=J_{S(x)}^{-1} \Phi(x, n) J_{x} .
$$

В связи с этой эквивалентностью возникает несколько задач. Например, верно ли, что эргодическое косое произведение $S \times\left\{T_{x}\right\}$ эквивалентно такому $S \times\left\{P_{x}\right\}$, что множество $\left\{P_{x}: x \in X\right\}$ является а) двухэлементным, b) конечным, с) счетным? Этот вопрос сформулировал В. И. Оселедец.

Настоящая заметка касается другой задачи: для какого множества $F \subset \operatorname{Aut}(\mu)$ косое произведение $S \times\left\{T_{x}\right\}$ эквивалентно $S \times\left\{P_{x}\right\}$, такому, что $\left\{P_{x}\right\} \subset F$. В качестве $F$ можно взять семейство всех автоморфизмов периода $m(m \geqslant 2)$. В заметке рассматривается случай $m=2$.

Ниже $E$ обозначает тождественный автоморфизм. Напомним, что автоморфизм $S$ называется эргодическим, если любое $S$-инвариантное измеримое множество имеет меру 0 или 1.

Теорема. Пусть $S \in \operatorname{Aut}(\mu)$ - эргодический автоморфизм пространства Лебега $(X, \mathscr{B}, \mu)$ и задано косое произведение $R=S \times\left\{T_{x}\right\}$. Тогда $R$ эквивалентно некоторому $\bar{R}=S \times\left\{P_{x}\right\}$, удовлетворяющему условию $P_{x}^{2}=E$.

В доказательстве используется следующее утверждение.

Лемма. Пусть $\left\{T_{x}: x \in X\right\}$ - измеримое семейство автоморфизмов пространства Лебега $(X, \mathscr{B}, \mu)$. Тогда найдутся измеримье семейства $\left\{F_{x}\right\}$, $\left\{G_{x}\right\}$ u $\left\{H_{x}\right\}$, maкue, ито $T_{x}=H_{x} G_{x} F_{x}$ u $F_{x}^{2}=G_{x}^{2}=H_{x}^{2}=E$.

\footnotetext{
${ }^{1}$ Работа поддержана Международным научным фондом, грант M1E000.
} 
ДокАЗАТЕЛЬСТво ЛЕммы. В [5] показано, что автоморфизм $\bar{T}$ полной булевой алгебры есть произведение трех инволюций из полной группы $[\bar{T}]$. Положим $\bar{T}=E \times\left\{T_{x}\right\}$; тогда для некоторых $\bar{F}, \bar{G}, \bar{H} \in[\bar{T}]$ имеем

$$
\bar{T}=\bar{H} \bar{G} \bar{F}, \quad \bar{F}^{2}=\bar{G}^{2}=\bar{H}^{2}=E .
$$

В силу определения полной группы траектории точек пространства $X \times Y$ под действием преобразования $\bar{T}$ являются инвариантными относительно преобразований $\bar{F}, \bar{G}, \bar{H} \in[\bar{T}]$. Так как множества вида $\{x\} \times Y$ инвариантны относительно $[\bar{T}]$, они будут инвариантными относительно $\bar{F}, \bar{G}, \bar{H}$. Таким образом, требуемые измеримые семейства автоморфизмов получаются из представлений

$$
\bar{F}=E \times\left\{F_{x}\right\}, \quad \bar{G}=E \times\left\{G_{x}\right\}, \quad \bar{H}=E \times\left\{H_{x}\right\} .
$$

ЗАМЕЧАНИЕ. В эргодической теории известны конструкции автоморфизмов, не сопряженных своему обратному (см. [2-4]). Такие автоморфизмы не могут быть композициями двух инволюций. В связи с этим отметим, что в полной группе $[T]$ эргодический автоморфизм $T$ и автоморфизм $T^{-1}$ не сопряжены. Действительно, пусть $S T=T^{-1} S$ и каждая траектория $O_{x}=\left\{\ldots, T^{-1}(x)\right.$, $x, T(x), \ldots\}$ инвариантна относительно автоморфизма $S$. Тогда для каждой траектории $O_{x}$ можно указать единственную точку $y\left(O_{x}\right) \in O_{x}$, такую, что $S\left(y\left(O_{x}\right)\right)=y\left(O_{x}\right)$ или $S\left(y\left(O_{x}\right)\right)=T\left(y\left(O_{x}\right)\right)$. Это легко следует из того, что левый и правый сдвиги на $\mathbb{Z}$ может сопрягать только симметрия $\sigma$ вида $\sigma(z)=$ $a-z$. По построению множество $Y=\bigcup_{x \in X}\left\{y\left(O_{x}\right)\right\}$ измеримо. Но, с другой стороны, подобно множеству Витали, множество $Y$ является неизмеримым. Если $\mu(Y)=0$, то $\mu(X)=0$, а $\mu(Y)>0$ влечет за собой $\mu(X)=\infty$. Следовательно, $T$ и $T^{-1}$ не сопряжены.

ДОКАЗАТЕЛЬСТВО ТЕОРЕМЫ. Ниже будет определено косое произведение $\widetilde{R}=S \times\left\{\widetilde{T}_{x}\right\}$, эквивалентное заданному $R$ и некоторому $\bar{R}=S \times\left\{\widetilde{P}_{x}\right\}, P_{x}^{2}=E$. Так как автоморфизм $S$ апериодичен, для некоторого множества $U \in \mathscr{B}$ выполнены условия $\mu(U)>0, \mu(U \cap S U)=\mu\left(U \cap S^{2} U\right)=0$. Положим $U_{0}=U$ и определим по индукции $U_{k+1}=S U_{k} \backslash U_{0}$. Объединение всех $U_{k}$ инвариантно относительно $S$; поэтому в силу эргодичности автоморфизма $S$ получим, что это объединение совпадает $\bmod 0 \mathrm{c} X$. На множестве $U_{0}$ определим функцию возвращения $r(x)$ :

$$
r(x)=\min \left\{i>0: T^{i}(x) \in U_{0}, x \in U_{0}\right\} .
$$

Положим $\Phi(x, n)=T_{S^{n-1}(x)} \cdots T_{S(x)} T_{x}$ и определим семейство автоморфизмов $\left\{\widetilde{T}_{x}\right\}$ следуюшим образом:

$$
\widetilde{T}_{x}= \begin{cases}\Phi(x, r(x)), & x \in U_{0}, \\ E, & x \in X \backslash U_{0} .\end{cases}
$$

Косые произведения $\widetilde{R}=S \times\left\{\widetilde{T}_{x}\right\}$ и $R$ эквивалентны. Действительно, пусть

$$
J_{x}^{-1}= \begin{cases}\Phi\left(x, r\left(T^{-k}(x)\right)-k\right), & x \in U_{k}, k>0, \\ E, & x \in U_{0} .\end{cases}
$$


Тогда выполнено (1). Пользуясь леммой, получаем $\widetilde{T}_{x}=H_{x} G_{x} F_{x}$.

Определим семейство инволюций $\left\{P_{x}\right\}, x \in X$, следующим образом:

$$
P_{x}= \begin{cases}F_{x}, & x \in U_{0}, \\ G_{S^{-1}(x)}, & x \in U_{1}, \\ H_{S^{-2}(x)}, & x \in U_{2}, \\ E, & \text { для остальных } x \in X .\end{cases}
$$

Заметим, что косое произведение $\bar{R}=S \times\left\{P_{x}\right\}$ эквивалентно $\widetilde{R}$. Действительно, если по $\bar{R}$ построить $\widetilde{\bar{R}}$ (аналогично тому, как по $R$ строилось $\widetilde{R}$ ), то получим $\widetilde{\bar{R}}=\widetilde{R}$. Так как $R$ эквивалентно $\widetilde{R}$, по транзитивности получим эквивалентность косых произведений $R$ и $\bar{R}$.

Автор благодарен В. И. Оселедцу, Б. М. Гуревичу и А. М. Степину за вопросы и обсуждения.

\title{
ЛитератУРА
}

1. Вериик А. М., Корнфельд И. П., Синай Я. Г. Общая эргодическая теория групп преобразований с инвариантной мерой. Совр. проблемы математики. Фундаментальные направления. Итоги науки и техники, Т. 2, ВИНИТИ, М. (1985), с. 5-111. 2. Оселедеи В. И. Функц. анализ и его прил., 5, вып. 3, 75-79 (1971). 3. Anzai H. Osaka Math. J., 3, No. 1, 83-99 (1951). 4. Малкин C. А. Изв. вузов, Математика, № 6, 69-74 (1968). 5. Рыжиков В. В. Матем. заметки, 54, вып. 2, 79-84 (1993).

Московский государственный университет им. М. В. Ломоносова

\section{Неунитарная часть тензорного произведения двух представлений группы $S O_{0}(n-1,1)$, одно из которых унитарно в индефинитной метрике}

\author{
(c) 1996. Ш. Ш. Султанов
}

Рассмотрим тензорное произведение $T_{\nu} \otimes T_{\sigma}=T$ представлений группы $S O_{0}(n-1,1)=G$, где $T_{\nu}$ - представление основной серии, $\nu=(2-n+2 i \rho) / 2$, $\rho \in \mathbb{R}$, а $T_{\sigma}$ унитарно в $\Pi_{r(m)}$-метрике, $2-n-m<\sigma<3-n-m, m \in \mathbb{N}$ (о представлениях, унитарных в индефинитной метрике, см. в [1]). Разложение тензорного квадрата представления дополнительной серии $T_{\sigma},-1<\sigma<0, n=3$, исследовано в [2], другие унитарные случаи для $n=3$ рассмотрены в [3-5]. Значительно меньше известно о неунитарных тензорных произведениях представлений групп Ли. Некоторые тензорные произведения, унитарные в $\Pi_{\infty}$-метрике, изучены в работах [6-8].

Мы будем использовать обозначения из [9] (гл. Х) с некоторыми упрощениями; например, вместо $T^{n, s}$ из [9] будем писать $T_{s}$. Для расширений представлений 